inflammatory diseases as compared to healthy secondary lymphoid organs. The striking link with microsatellite instability and unfavorable tumor features suggest a potential clinical utility of assessing $\mathrm{Ki} 67^{+} \mathrm{CD} 8^{+}$in colorectal cancer.

Disclosure Information K. Möller: None. M. Lennartz: None. R. Abu-Hashem: None. N.C. Blessin: None. T. Mandelkow: None. E. Bady: None. C. Hube-Magg: None. R. Simon: None. G. Sauter: None. C. Fraune: None. T.S. Clauditz: None. F. Büscheck: None. A.M. Luebke: None.

\section{P03.07 FAST AUTOMATED MICROFLUIDIC-BASED MULTIPLEXED IMMUNOFLUORESCENCE FOR TUMOR MICROENVIRONMENT ANALYSIS}

${ }^{1}$ A Kehren*, ${ }^{2}$ MG Procopio, ${ }^{1} B$ Pelz, ${ }^{1} Z$ Siddiqui, ${ }^{3} \mathrm{~K}$ Roman, ${ }^{1} \mathrm{~S}$ Adnane, ${ }^{1} \mathrm{~S}$ Brajkovic, ${ }^{3} \mathrm{C}$ Hoyt, ${ }^{1}$ DG Dupouy, ${ }^{2} \mathrm{~A}$ Soltermann. ' Lunaphore Technologies SA, Lausanne, Switzerland; ${ }^{2}$ Institut für Klinische Pathologie Universitätsspital Zürich, Zürich, Switzerland; ${ }^{3} A k o y a$ Biosciences, Hopkinton, MA, USA

\subsection{6/jitc-2020-ITOC7.47}

Background Immuno-oncology and targeted molecular therapies have acquired a central role in the treatment of multiple cancers. Consequently, high-throughput biomarker analysis and tumor immune profiling have seen an increased demand. Multiplexed immuno-assays are a powerful tool to address these needs, but still time- and resource-consuming. Our goal is to develop a fast and automated high-plex fluorescent immunostaining procedure, using a microfluidic-based device, that can be easily implemented as routine assay.

Materials and Methods Protocol optimization has been performed on FFPE sections of human tonsil. Slides were manually deparaffinized before being entirely processed (antigenretrieval, staining, elution and counterstaining) by Lunaphore's autostainer, LabSat ${ }^{\mathrm{TM}}$. The $\mathrm{OPAL}^{\circledR}$ tyramide signal amplification (TSA) system was used as detection method. Signal analysis was done on Mantra ${ }^{\circledR}$ workstation. The 6-plex panel was composed of FoxP3, PD-L1, PD-1, CD68, CD8 and pan-CK, plus DAPI counterstaining. Protocols were subsequently transferred on NSCLC representative specimens and finally assessed on a TMA cohort.

Results Our platform allowed to reduce drastically the incubation times due to active transport of reagents across the tissue. Thereby, the automated 6-plex assay could be performed in less than $4 \mathrm{~h} 30 \mathrm{~min}$, within the timeframe of a single IHC standard assay. Protocol optimization resulted in high signalto-background ratio for each marker and removal of previous step antibodies over 99\%. LabSat ${ }^{\mathrm{TM}}$ also guaranteed remarkable signal uniformity, even over large tissue sections with less than $10 \%$ signal gradient over $1 \mathrm{~cm}$. On NSCLC samples, the detected pattern and expression level for all six biomarkers were comparable to the standard chromogenic stainings performed with standard automated tissue stainer.

Conclusions LabSat ${ }^{\mathrm{TM}}$ autostainer enables multistaining runs in a timely manner, opening the perspective of rapid simultaneous detection of multiple markers in their morphological context on a routine-based approach. This versatile analysis tool can offer a better and more quantitative understanding of tumor heterogeneity and microenvironmental interactions, allowing advances in targeted therapy for lung cancer as well as broader spectrum of malignancies.

Disclosure Information A. Kehren: A. Employment (full or part-time); Significant; Lunaphore Technologies SA. C. Other Research Support (supplies, equipment, receipt of drugs or other in-kind support); Significant; Akoya Biosciences. M.G. Procopio: B. Research Grant (principal investigator, collaborator or consultant and pending grants as well as grants already received); Significant; KTI grant (Schweiz). B. Pelz: A. Employment (full or part-time); Significant; Lunaphore Technologies SA. C. Other Research Support (supplies, equipment, receipt of drugs or other in-kind support); Significant; Akoya Biosciences. Z. Siddiqui: A. Employment (full or part-time); Modest; Lunaphore Technologies SA. K. Roman: A. Employment (full or part-time); Significant; Akoya Biosciences. S. Adnane: A. Employment (full or part-time); Modest; Lunaphore Technologies SA. S. Brajkovic: A. Employment (full or part-time); Significant; Lunaphore Technologies SA. C. Hoyt: A. Employment (full or part-time); Significant; Akoya Biosciences. D.G. Dupouy: A. Employment (full or part-time); Significant; Lunaphore Technologies SA. E. Ownership Interest (stock, stock options, patent or other intellectual property); Significant; Lunaphore Technologies SA. A. Soltermann: A. Employment (full or part-time); Significant; Institut für Klinische Pathologie Universitätsspital Zürich.

\section{P03.08 OMENTAL FAT IN OVARIAN CANCER POTENTIALLY INDUCES LYMPHANGIOGENESIS}

${ }^{1}$ BMA Lenoir*, ${ }^{2}$ V Starrach, ${ }^{2} \mathrm{D}$ Ferber, ${ }^{1} \mathrm{M}$ Suarez-Carmona, ${ }^{3} \mathrm{~S}$ Schott, ${ }^{1} \mathrm{I}$ Zörnig, ${ }^{1,2} \mathrm{D}$ Jäger, ${ }^{1,2} \mathrm{~N}$ Halama. ${ }^{1} D K F Z$, Heidelberg, Germany; ${ }^{2} N C T$, Heidelberg, Germany; ${ }^{3}$ University hospital, Heidelberg, Germany

\subsection{6/jitc-2020-ITOC7.48}

Background Ovarian cancer metastasis occurs by direct multifocal seeding in the peritoneum as well as by migration through the lymphatic system. High grade ovarian carcinoma patients present with distant metastases. Significant risk factors for the development of those are stage, grade, and lymph node involvement. An increase of the number of lymphatic vessels is shown in ovarian tumors and these vessels seem implicated in tumor progression. While the tropism of ovarian cancer cells for fat is well described, the potential impact of a fatty microenvironment on the dissemination of tumor cells via lymphatic vessels has, to our knowledge, never been investigated yet. In this study, we examined the effect of omental fat on lymphangiogenesis in ovarian carcinoma.

Materials and Methods To examine the effect of omental fat on lymphangiogenesis in OC we used a cohort of 80 human specimens. We analysed lymphatic vessels histologically with D2-40 and Lyve-1 markers. We also developed a healthy fat tissue explant culture model and treated explants with ascites of patient with OC before analysis. We analysed by fluorescence stainings the co expression of adipose derived stem cells (ASCs) and lymphatic markers in these explants.

Results We observed a higher density of tumor-associated vessels, especially lymphatic vessels in OC in contact with the omentum; mainly localized along the adipose tissue. We also measured a higher secretion of VEGF-C in tissues with fat compared to tissues without fat. Healthy fat tissues treated with ascites show an increase of number of ASCs, some of them express lymphatic markers such as D2-40 and Lyve-1. In a clinical trial of patients with OC treated by Bevacizumab, we observed a decrease of the number of lymphatic vessels in correlation with a decrease of the inflammation around the fat tissue.

Discussion We saw an increase in the number of lymphatic vessels in ovarian carcinoma infiltrating fat. These vessels are 
principally distributed around the fat. We also observed an increase of proliferating ASC expressing lymphatic marker in fat explants treated with ascites. In a clinical trial of patients treated with Bevacizumab, we see a decrease of the lymphatic vessels. This decrease is linked with a decrease in the number of Inflammatory cells. These results together show that the fat tissue can play an important role in the lymphangiogenesis in the ovarian carcinoma. Furthermore, in the dissemination of metastasis through the body. We will next investigate the mechanisms underlying this phenomenon and try to understand all factors implicated in this process.

Disclosure Information B.M.A. Lenoir: None. V. Starrach: None. D. Ferber: None. M. Suarez-Carmona: None. S. Schott: None. I. Zörnig: None. D. Jäger: None. N. Halama: None.

\section{P03.09 ABSTRACT WITHDRAWN}

\section{P03.10 PREVALENCE AND PROGNOSTIC ROLE OF FOXP3 ${ }^{+}$REGULATORY T LYMPHOCYTES IN CANCER. A TISSUE MICROARRAY STUDY ON >20'000 CANCERS}

T Mandelkow*, E Bady, NC Blessin, C Hube-Magg, R Simon, G Sauter, C Fraune, M Lennartz, K Möller, SA Weidemann, AM Luebke, D Höflmayer, F Büscheck. University Medical Center Hamburg-Eppendorf, Hamburg, Germany

\subsection{6/jitc-2020-ITOC7.49}

Background Regulatory FoxP3 ${ }^{+}$lymphocytes function as suppressors of T-cell activity. The clinical impact of high FoxP3 ${ }^{+}$ cell density in cancers is not fully understood, as some studies have linked high $\mathrm{FoxP}^{+}$cell density to good prognosis and others to poor prognosis in tumor cohorts with associated clinical data. While some data suggest that these variable data are due to biological differences between tumor entities, it is also possible that methodological differences have caused these discrepancies. This study was undertaken to analyze the density of $\mathrm{FoxP}^{+}$cells in various different cancer types by employing standardized methods.

Materials and Methods Tissue microarrays and large sections made from $>20,000$ prostate, breast, colorectal, ovarian, pancreatic, bladder and stomach cancers were analyzed together with various normal and inflamed tissues by conventional brightfield FoxP3 immunohistochemistry. Samples were also analyzed by fluorescent multiplex immunohistochemistry to assess the fraction of $\mathrm{Ki}_{6} 7^{+} \mathrm{FoxP}^{+}$cells.

Results Our results indeed suggested a variable role of $\mathrm{FoxP}^{+}$ cells in different tumor types. High $\mathrm{FoxP}^{+}$density was linked to high Gleason grade $(\mathrm{p}=0.0003)$ and early biochemical recurrence $(\mathrm{p}<0.0001)$ in 16923 prostate cancers, but to low tumor stage $(\mathrm{p}=0.027)$ and prolonged survival $(p=0.0029)$ in 1341 breast cancers, and to low tumor stage $(\mathrm{p}<0.0001)$ in 744 colorectal cancers. No significant associations were found to tumor phenotype in 549 ovarian, 574 pancreatic, 549 bladder and 346 stomach cancers. Multiplex fluorescence IHC analysis of FoxP3 and Ki67 revealed comparable fractions of proliferating $\mathrm{FoxP}^{+}$cells in healthy tissues (average 12.3\%, range 5.8-18.5\%) and inflammatory conditions (average $7.6 \%$, range 2.6-17.2\%). Interestingly, the rate of $\mathrm{Ki} 67^{+} \mathrm{FoxP}^{+}$cells was markedly higher in 36 bladder cancers (average 14.2\%, range 0-49.3\%) suggesting active expansion of $\mathrm{FoxP}^{+}$cells in cancer.
Conclusions Our data demonstrate an inverse prognostic impact of the $\mathrm{FoxP}^{+}$cell density in prostate and breast cancers. The increased proliferation rate of immune-regulatory $\mathrm{FoxP}^{+}$cells in some bladder cancer is interesting in the light of the variable response of these tumors to immune checkpoint inhibitors.

Disclosure Information T. Mandelkow: None. E. Bady: None. N.C. Blessin: None. C. Hube-Magg: None. R. Simon: None. G. Sauter: None. C. Fraune: None. M. Lennartz: None. K. Möller: None. S.A. Weidemann: None. A.M. Luebke: None. D. Höflmayer: None. F. Büscheck: None.

\section{P03.11 EXPLORING TUMOR-INTRINSIC FACTORS REGULATING THE RECRUITMENT OF MYELOID-DERIVED SUPPRESSOR CELLS (MDSC) IN PANCREATIC DUCTAL ADENOCARCINOMA}

${ }^{1} \mathrm{C}$ Rambuscheck*, ${ }^{1} \mathrm{P}$ Metzger, ${ }^{1} \mathrm{C}$ Hörth, ${ }^{2} \mathrm{R}$ Hennel, ${ }^{3} \mathrm{~S}$ Bärthel, ${ }^{3} \mathrm{C}$ Falcomatà, ${ }^{2} \mathrm{~K}$ Lauber, ${ }^{1} \mathrm{~S}$ Endres, ${ }^{3} \mathrm{D}$ Saur, ${ }^{1} \mathrm{M}$ Schnurr, ${ }^{1} \mathrm{LM}$ König. ${ }^{1}$ Center of Integrated Protein Science Munich (CIPSM) and Division of Clinical Pharmacology, University Hospital, LMU, Munich, Germany; ${ }^{2}$ Department of Radiation Oncology, University Hospital, LMU, Munich, Germany; ${ }^{3}$ Institute of Translational Cancer Research and Experimental Cancer Therapy, Klinikum rechts der Isar, Technische Universität München, Munich, Germany

\subsection{6/jitc-2020-ITOC7.50}

Background Pancreatic Ductal Adenocarcinoma (PDAC) has very poor 5-year overall survival rate. Despite the encouraging effect of immunotherapy in other cancer types, clinical benefit in PDAC patients remains limited. One of the reasons for the lack of success is the immunosuppressive tumor microenvironment (TME), which is maintained by myeloidderived suppressor cells (MDSC) and tumor-associated macrophages. High MDSC infiltration is associated with a poor survival in PDAC patients. Our project aims at identifying tumor-driven chemokines that influence recruitment of MDSC and establishment of the immunosuppressive tumor microenvironment.

Materials and Methods 45 PDAC cell lines generated from spontaneous tumors of genetically-modified mice harboring the characteristic driver mutations Kras ${ }^{G 12 D}$ or PIK3CA ${ }^{H 1047 R}$ were analyzed for their expression levels of CXCL1, CCL2, G-CSF and GM-CSF by qRT-PCR. In order to study the relationship between the chemokine/cytokine profile and the immune cell infiltration, selected tumor cell lines were implanted orthotopically in C57BL6 mice. Three weeks after inoculation blood, spleen and tumor were isolated and organ specific immune cell infiltration was analyzed by flow cytometry. To further characterize tumor-secreted factors tumor conditioned medium was generated and the concentration of 33 chemokines was analyzed in a multiplex assay. The chemokine levels were correlated with migratory capacity of splenic MDSC measured in an ex vivo chemotaxis assay.

Results CXCL1 significantly enhanced migration of polymorphonuclear MDSC (PMN-MDSC) in vitro, while migration of monocytic MDSC (M-MDSC) was predominantly skewed towards CCL2. Three weeks after tumor inoculation, MDSC populations in blood and spleen were expanded. Most intriguingly, PDAC cell lines with high CXCL1 or CCL2 levels in vitro showed significantly enriched intratumoral accumulation of PMN-MDSC and M-MDSC, respectively, suggesting that tumor-intrinsic chemokine secretion and not factors from the tumor stroma determined MDSC infiltration. The ex vivo chemotaxis assays revealed additional factors that modulate migration of MDSC into the TME. 